\title{
Crystal structure of human Gadd45y reveals an active dimer
}

\author{
Wenzheng Zhang ${ }^{1,2,3^{*}}$, Sheng $\mathrm{Fu}^{1 *}$, Xuefeng Liu ${ }^{4}$, Xuelian Zhao ${ }^{4}$, Wenchi Zhang ${ }^{1}$, Wei Peng ${ }^{1}$, Congying $\mathrm{Wu}^{3}$ \\ Yuanyuan $\mathrm{Li}^{3}$, Xuemei Li ${ }^{1}$, Mark Bartlam ${ }^{1,2}$, Zong-Hao Zeng ${ }^{1 凶}$, Qimin Zhan $^{4}$, Zihe Rao ${ }^{1,2,3}$ \\ ${ }^{1}$ National Laboratory of Biomacromolecules, Institute of Biophysics, Chinese Academy of Sciences, Beijing 100101, China \\ ${ }^{2}$ Tianjin Key Laboratory of Protein Science, College of Life Sciences, Nankai University, Tianjin 300071, China \\ ${ }^{3}$ Laboratory of Structural Biology, Tsinghua University, Beijing 100084, China \\ ${ }^{4}$ State Key Laboratory of Molecular Oncology, Cancer Institute, Chinese Academy of Medical Sciences and Peking Union \\ Medical College, Beijing 100021, China \\ $\square$ Correspondence: zzh@ibp.ac.cn (Z.-H. Zeng), zhanqimin@pumc.edu.cn (Q. Zhan) \\ Received August 23, 2011 Accepted September 19, 2011
}

Erratum to: Protein Cell 2011, 2(10): 814-826

DOI 10.1007/s13238-011-1090-6

Due to typesetting errors, "gadd45" should be "Gadd45y" in the following places: the article title, the $2^{\text {nd }}, 4^{\text {th }}$ and $5^{\text {th }}$
Gadd45 in the ABSTRACT, the KEYWORDS, the first subtitle of "RESULTS AND DISCUSSION" section, the legend of Figure 1, the "data collection and processing" of MATERIALS AND METHODS section.

The online version of the original article can be found at http://dx.doi.org/10.1007/s13238-011-1090-6

\footnotetext{
*These authors contributed equally to the work.
} 